\title{
Accelerating Electrochemical Synthesis through Automated Flow: Efficient Synthesis of Chalcogenophosphites
}

\author{
Nasser Amri \\ Thomas Wirth* \\ School of Chemistry, Cardiff University, Park Place, Cardiff, \\ CF10 3AT, UK \\ wirth@cf.ac.uk \\ Published as part of the Cluster Integrated Synthesis Using \\ Continuous-Flow Technologies
}

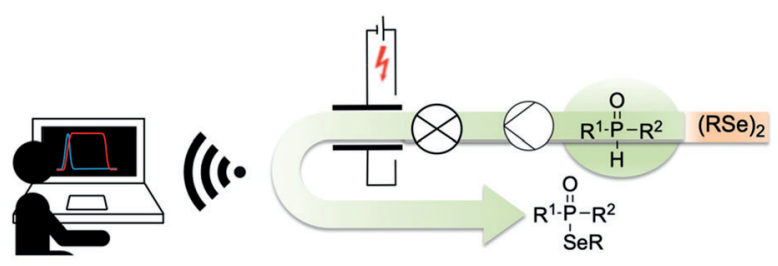

The synthesis of selenium-phosphorus or sulfur-phosphorus bonds has been studied with different chemical methods but often expensive metals or toxic reagents are used. ${ }^{3}$ Phosphorus can form bonds with various nucleophiles in electrochemical batch reactions using supporting electrolytes such as $\mathrm{Bu}_{4} \mathrm{NI}^{4,5} \mathrm{Bu}_{4} \mathrm{NBr},{ }^{6} \mathrm{KI},{ }^{7}$ and $\mathrm{DMMI}^{8}$ as halogen-containing mediators with electrodes such as platinum, and metal catalyst such as $\mathrm{Cu}^{9}{ }^{9}$ with typically long reaction times. Very recently, such electrochemical methods have been applied in the selective formation of phosphorus-sulfur ${ }^{4}$ and phosphorus-selenium ${ }^{6}$ bonds. Lee, Cai, and co-authors used stoichiometric amounts of $\mathrm{Bu}_{4} \mathrm{NI}$ and $\mathrm{Bu}_{4} \mathrm{NBr}$ electrolytes and Pt electrodes with reaction times of 6-7 hours. While batch electrochemical methods are standard protocols, continuous-flow syntheses ${ }^{10}$ and flow electrochemical reactions ${ }^{11}$ have recently emerged as highly beneficial additions to the portfolio of organic chemists in academia and industry. Electrochemistry integrated in flow reactions has not yet been explored to obtain phosphorusfunctionalised chalcogen derivatives. In continuation of our efforts towards the development of tools and techniques to carry out flow transformations, we have recently disclosed the first use of an integrated flow electrochemical reactor in an automated way. ${ }^{12}$ In this work we apply the same methodology for the efficient synthesis of phosphorus-sulfur and phosphorus-selenium bonds.

The Vapourtec automated flow system with the integrated ion electrochemical microflow reactor has unique features which accelerate the reaction process and minimise the required manpower. The system is able to self-load the required chemicals, collect the reaction output, autoclean the system while allowing exact temperature, flow rate, and current control in each reaction. Furthermore, it can be operated remotely from the office or other locations with internet connection. The only limitation faced in this

Figure 1 Bioactive molecules with P-Se and P-S bonds 
system is the number of accessible loading and collection vials. A schematic representation of the system is shown in Figure 2.

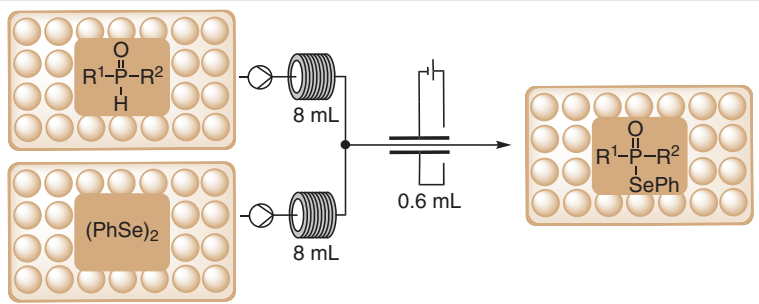

Figure 2 Automated flow electrochemical synthesis of chalcogenophosphites

Initially, the reaction between diethylphosphite (1) and diphenyl diselenide (2) in acetonitrile was investigated using different reaction conditions, electrode materials, and electrolytes. Interestingly, the reaction does not proceed without electrolyte despite the close distance of the electrodes in the flow reactor $(0.5 \mathrm{~mm})$ indicating a participation of the electrolyte as a mediator in this transformation.

In this reaction, initially different electrolytes such as $\mathrm{Et}_{4} \mathrm{NI}, \mathrm{Et}_{4} \mathrm{NBr}, \mathrm{Et}_{4} \mathrm{NCl}, \mathrm{Bu}_{4} \mathrm{NBF}_{4}$, and $\mathrm{Bu}_{4} \mathrm{NClO}_{4}$ were screened and among these $\mathrm{Et}_{4} \mathrm{NCl}$ showed the highest yield of $97 \%$ (Table 1 , entries $1-5$ ). To reduce the time of reaction, the flow rate was increased from $1.0 \mathrm{~mL} / \mathrm{min}$ up to $1.8 \mathrm{~mL} / \mathrm{min}$. At $1.2 \mathrm{~mL} / \mathrm{min}$ still $97 \%$ yield was obtained but at higher flow rates lower yields were observed (Table 1, entries 6-8). Acetonitrile was already the solvent of choice, other solvents such as dichloromethane, methanol, or dimethylformamide resulted in decreased yields (Table 1, entries 9-11). Different electrode combinations were screened but these were all less encouraging. A decrease in the phosphite concentration led to reduced yields of the product (see the Supporting Information). The optimal reaction conditions with the highest space-time yield are shown in entry 6 of Table 1. These conditions have now been applied to the automated synthesis of a large variety of products in order to investigate the scope and general applicability of this electrochemical methodology (Scheme 1). ${ }^{13}$

Phosphite derivatives such as ethoxy (3a), methoxy (3b), n-butoxy (3c), isopropoxy (3d), 2-ethylhexyl (3e), and benzyl (3f) provided the products in good yields. A cyclic phosphite was investigated and formed the product $\mathbf{3 g}$ with $84 \%$ isolated yield. Similarly, the phenoxy-substituted product 3 h was formed in an excellent yield of 95\%. Among different phosphine oxide derivatives, the phenyl (3i), tolyl (3j), 3,5-dimethylbenzene (3k), and 2-naphthyl (31) substituted products were isolated in good yields. Also unsymmetrical compounds bearing ethoxy and phenyl substituents provided the product $\mathbf{3 m}$ in $80 \%$ yield. Cyclic derivative 3n was formed in $86 \%$ yield. Similar to diphenyl diselenide, other dichalcogenides such as dibenzyl diselenide and diphenyl disulfide could be employed to give phosphorosele-
Table 1 Reaction of 1 and $\mathbf{2}$ to O,O-Diethyl Se-Phenyl Phosphoroselenoate (3a) Using a Flow Electrochemical Reactor ${ }^{\mathrm{a}}$

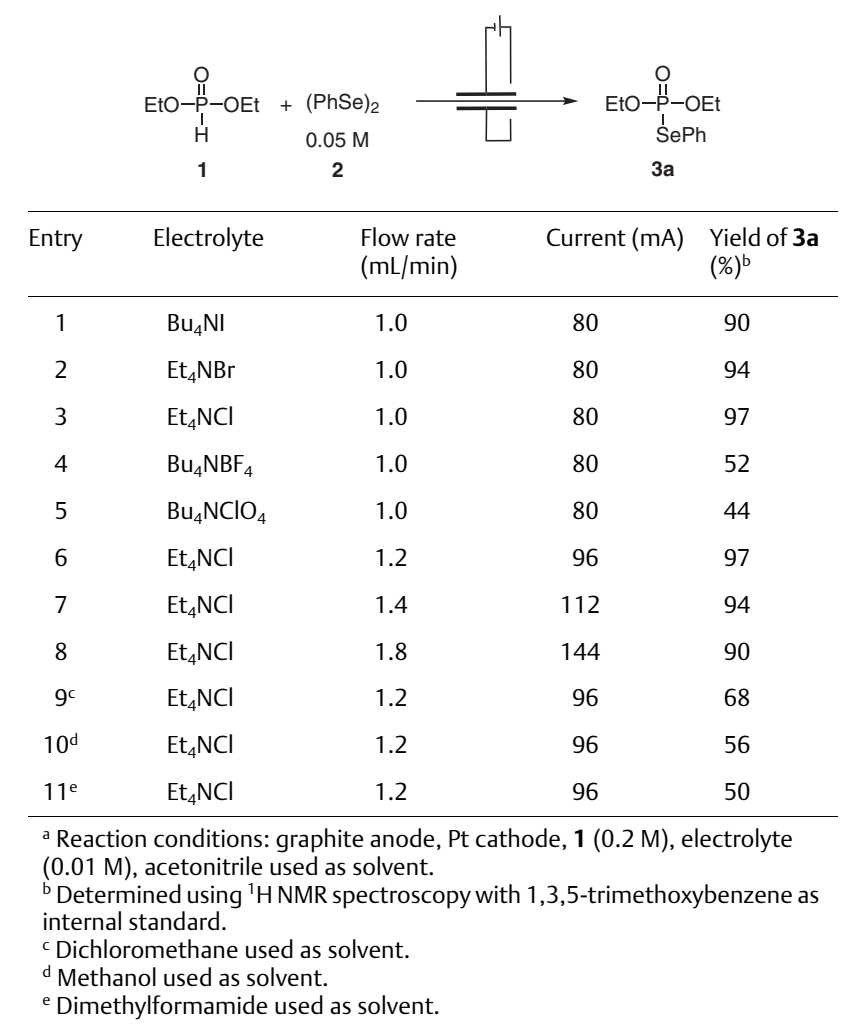

noates 3o-r and phosphorosulfonates $3 \mathbf{s}-\mathbf{u}$, but the yields of the phosphorosulfonates were found to be slightly lower than the yields of the corresponding phosphorosele-

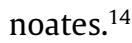

In order to get some insight into the mechanism, we studied the electrooxidation properties of each compound. From cyclic voltammogram (CV) experiments, diethyl phosphite shows no oxidation peak and diphenyl diselenide shows two oxidation peaks at $1.46 \mathrm{~V}$ and $1.8 \mathrm{~V}$ ( $\mathrm{vs} \mathrm{Ag} / \mathrm{AgCl}$ ) while $\mathrm{Et}_{4} \mathrm{NCl}$ showed an obvious oxidation peak at $1.41 \mathrm{~V}$ (vs $\mathrm{Ag} / \mathrm{AgCl}$ ) indicating that $\mathrm{Et}_{4} \mathrm{NCl}$ is earlier oxidised (see Figure S3, Supporting Information).

Based on previous literature reports ${ }^{4-6,8,15}$ and our initial studies, the proposed mechanism involved in the selenenylation of phosphites is shown in Scheme 2. The diselenide $\mathbf{2}$ is reduced at the cathode to generate the radical anion $\mathbf{I}$, which then reacts the phosphite to generate the selenophosphite $\mathbf{3}$ and the radical anion II. This intermediate II is then oxidised to the selenyl radical III which dimerises to $\mathbf{2}$ and a proton, which will be finally reduced to hydrogen at the cathode. Other mechanisms in the literature suggest the involvement of elemental halides $s^{4,6}$ or halide radicals ${ }^{8}$ generated from the electrolyte. This cannot be excluded here, but the fact that the reaction proceeds also without halide anions (Table 1, entries 4 and 5) suggest that a different mechanism must also be operating here. 


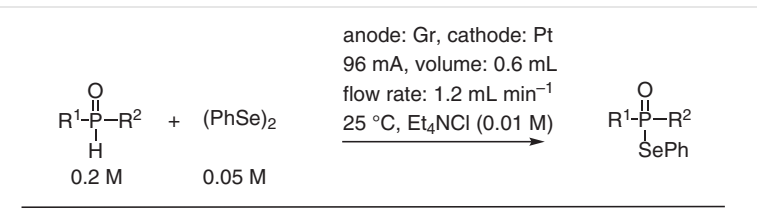

\begin{tabular}{|c|c|c|c|}
\hline $\begin{array}{l}\text { O } \\
\text { O-P-OEt } \\
\text { SePh }\end{array}$ & $\begin{array}{c}\text { O } \\
\text { MeO-P P-OMe } \\
\text { SePh }\end{array}$ & $\begin{array}{c}\text { II } \\
{ }^{n} \mathrm{BuO}-\mathrm{P}_{-} \mathrm{O}^{n} \mathrm{Bu} \\
\text { SePh }\end{array}$ & $\begin{array}{c}\text { O } \\
\text { 'PrO-P-PiPr } \\
\text { SePh }\end{array}$ \\
\hline & & & \\
\hline
\end{tabular}$$
\begin{gathered}
\mathbf{3 f}(84 \%) \\
\mathbf{3 e}(79 \%)
\end{gathered}
$$

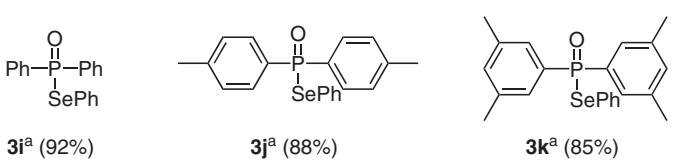

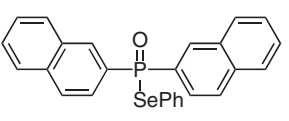

$3 \mathrm{l}^{\mathrm{a}}(83 \%)$

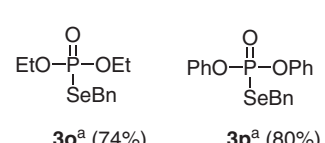

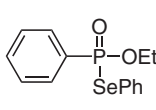

$3 \mathrm{~m}(80 \%)$

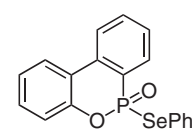

$3 \mathbf{n}^{\mathrm{a}}(86 \%)$

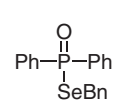

$3 \mathbf{q}^{\mathrm{a}}(78 \%)$

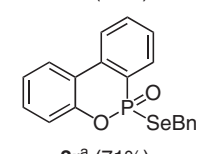

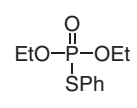

3s $(57 \%)$

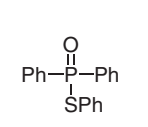

$3 \mathbf{t}^{\mathrm{a}}(66 \%)$

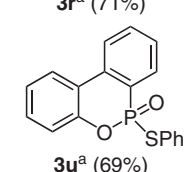

Scheme 1 Substrate scope for the electrochemical selenenylation and thiolation of phosphites. ${ }^{\text {a }} \mathrm{A}$ mixture of $\mathrm{CH}_{3} \mathrm{CN} / \mathrm{THF}$ (1:1) was used.

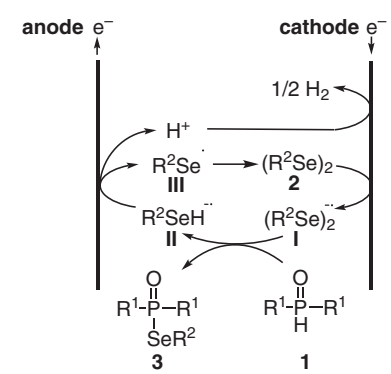

Scheme 2 Proposed mechanism for the electrochemical synthesis of selenophosphites

We have demonstrated that phosphites can be efficiently selenenylated in flow electrochemical reactors in moderate to good yields. The use of an automated flow system integrated with the electrochemical microreactor enabled a fast synthesis of different derivatives in a straightforward way. The scope and limitation of the method are illustrated with a broad range of substrates. Compared to previous procedures, this method is fast, inexpensive, metal-free, environmentally friendly, and automated. Future research in synthesising organic compounds in an automated electrochemical way is currently ongoing in our laboratory.

\section{Funding Information}

Support from Cardiff University and from the Chemistry Department, Jazan University, Saudi Arabia, are appreciated.

\section{Acknowledgment}

We thank D. Guthrie and M. Nuno, Vapourtec Inc. for many discussions and for providing the equipment and support for this research. Fruitful discussions with Dr. M. N. Khan, Cardiff University are gratefully acknowledged.

\section{Supporting Information}

Supporting information for this article is available online at https://doi.org/10.1055/s-0040-1707141.

\section{References and Notes}

(1) (a) Mugesh, G.; Du Mont, W. W.; Sies, H. Chem. Rev. 2001, 101, 2125. (b) Handbook of Organophosphorus Chemistry; Engel, R., Ed.; CRC Press: New York, 1992. (c) Quin, L. D. A Guide to Organophosphorus Chemistry; Wiley \& Sons: New York, 2000. (d) Han, L. B.; Tanaka, M. J. Am. Chem. Soc. 1996, 118, 1571. (e) Xie, R.; Zhao, Q.; Zhang, T.; Fang, J.; Mei, X.; Ning, J.; Tang, Y. Bioorg. Med. Chem. 2013, 21, 278. (f) Sova, M.; Kovač, A.; Turk, S.; Hrast, M.; Blanot, D.; Gobec, S. Bioorg. Chem. 2009, 37, 217. (g) Hua, G.; Woollins, J. D. Angew. Chem. Int. Ed. 2009, 48, 1368.

(2) (a) Morton, W. R.; Drance, S. M.; Fairclough, M. Am. J. Ophthalmol. 1969, 68, 1003. (b) Bhunia, S. K.; Das, P.; Jana, R. Org. Biomol. Chem. 2018, 16, 9243. (c) Masahiro, B. JP 53095946, 1978.

(3) (a) Kawaguchi, S. I.; Kotani, M.; Atobe, S.; Nomoto, A.; Sonoda, M.; Ogawa, A. Organometallics 2011, 30, 6766. (b) Wang, J.; Wang, X.; Li, H.; Yan, J. J. Organomet. Chem. 2018, 859, 75. (c) Zhang, X.; Shi, Z.; Shao, C.; Zhao, J.; Wang, D.; Zhang, G.; Li, L. Eur. J. Org. Chem. 2017, 1884. (d) Choudhary, R.; Singh, P.; Bai, R.; Sharma, M. C.; Badsara, S. S. Org. Biomol. Chem. 2019, 17, 9757. (e) Zhu, Y.; Chen, T.; Li, S.; Shimada, S.; Han, L. B. J. Am. Chem. Soc. 2016, 138, 5825.

(4) Li, C. Y.; Liu, Y. C.; Li, Y. X.; Reddy, D. M.; Lee, C. F. Org. Lett. 2019, $21,7833$.

(5) Deng, L.; Wang, Y.; Mei, H.; Pan, Y.; Han, J.J. Org. Chem. 2019, 84, 949.

(6) Guo, S.; Li, S.; Zhang, Z.; Yan, W.; Cai, H. Tetrahedron Lett. 2020, $61,151566$.

(7) Wang, Y.; Qian, P.; Su, J. H.; Li, Y.; Bi, M.; Zha, Z.; Wang, Z. Green Chem. 2017, 19, 4769.

(8) Dong, X.; Wang, R.; Jin, W.; Liu, C. Org. Lett. 2020, 22, 3062.

(9) Fu, N.; Song, L.; Liu, J.; Shen, Y.; Siu, J. C.; Lin, S. J. Am. Chem. Soc. 2019, 141, 14480. 
(10) (a) Jamison, T. F.; Koch, G. Flow Chemistry in Organic Synthesis; Thieme: Stuttgart, 2018. (b) Wirth, T. Microreactors in Organic Chemistry and Catalysis, 2nd ed; Wiley-VCH: Weinheim, 2013. (c) Yoshida, J. Flash Chemistry: Fast Organic Synthesis in Microsystems; Wiley-VCH: Weinheim, 2008.

(11) (a) Elsherbini, M.; Wirth, T. Acc. Chem. Res. 2019, 52, 3287. (b) Folgueiras-Amador, A. A.; Wirth, T. In Flow Chemistry in Organic Synthesis; Jamison, T. F.; Koch, G., Ed.; Thieme: Stuttgart, 2018. (c) Pletcher, D.; Green, R. A.; Brown, R. C. D. Chem. Rev. 2018, 118, 4573. (d) Atobe, M.; Tateno, H.; Matsumura, Y. Chem. Rev. 2018, 118, 4541. (e) Wiebe, A.; Gieshoff, T.; Möhle, S.; Rodrigo, E.; Zirbes, M.; Waldvogel, S. R. Angew. Chem. Int. Ed. 2018, 57, 5594.

(12) Amri, N.; Wirth, T. Synthesis 2020, 52, 1751.

(13) Experimental Conditions

The electrolysis was performed in an undivided cell using a Vapourtec ion electrochemical flow reactor (reactor volume $=$ $0.6 \mathrm{~mL}$, spacer $0.5 \mathrm{~mm}$ ) using a graphite $(\mathrm{Gr})$ anode and a platinum (Pt) cathode (surface area: $\mathrm{A}=12 \mathrm{~cm}^{2}$ ). A solution of phosphite $\left(0.2 \mathrm{M}\right.$ in $\left.\mathrm{CH}_{3} \mathrm{CN}\right)$ is placed in vial $\mathrm{A}$ and a mixture of diphenyl diselenide $(0.05 \mathrm{M})$ and $\mathrm{Et}_{4} \mathrm{NCl}(0.01 \mathrm{M})$ in $\mathrm{CH}_{3} \mathrm{CN}$ placed in vial B. Each solution was injected to $8 \mathrm{~mL}$ sample loop. The reactor temperature was set at $25{ }^{\circ} \mathrm{C}$ with a flow rate of 1.2

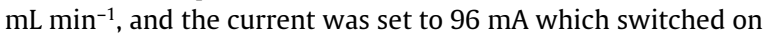
automatically. Then, both solutions were pumped into a PTFE coil (1 mm internal diameter) and mixed via a T-piece connected to a $30 \mathrm{~cm}$ PTFE coil before the inlet of the electrochemical rector. After reaching a steady state, the solution $(12 \mathrm{~mL})$ was collected at the reactor outlet automatically into a collection glass vial. The solvent was removed under vacuum. The crude product was purified by column chromatography (EtOAc/cyclohexane).

(14) Characterisation Data

\section{0,0-Diethyl Se-Phenyl Phosphoroselenoate (3a)}

$56 \mathrm{mg}\left(89 \%\right.$ yield ). ${ }^{1} \mathrm{H}$ NMR $\left(500 \mathrm{MHz}, \mathrm{CDCl}_{3}\right): \delta=7.69-7.60(\mathrm{~m}$, $2 \mathrm{H}), 7.38-7.29(\mathrm{~m}, 3 \mathrm{H}), 4.25-4.11(\mathrm{~m}, 4 \mathrm{H}), 1.30(\mathrm{td}, J=7.1,0.8$ $\mathrm{Hz}, 6 \mathrm{H}) .{ }^{13} \mathrm{C}$ NMR $\left(126 \mathrm{MHz}, \mathrm{CDCl}_{3}\right): \delta=135.7(\mathrm{~d}, J=3.7 \mathrm{~Hz})$, $129.6(\mathrm{~d}, J=1.3 \mathrm{~Hz}), 128.9$ (d, $J=2.5 \mathrm{~Hz}), 123.9(\mathrm{~d}, J=8.7 \mathrm{~Hz})$, $64.0(\mathrm{~d}, J=6.2 \mathrm{~Hz}), 16.05(\mathrm{~d}, J=7.5 \mathrm{~Hz}) .{ }^{31} \mathrm{P}$ NMR $(202 \mathrm{MHz}$, $\left.\mathrm{CDCl}_{3}\right): \delta=17.9$.

0,0-Dimethyl Se-Phenyl Phosphoroselenoate (3b)

$123 \mathrm{mg}$ (77\% yield). ${ }^{1} \mathrm{H}$ NMR $\left(500 \mathrm{MHz}, \mathrm{CDCl}_{3}\right): \delta=7.68-7.58$ (m, $2 \mathrm{H}), 7.39-7.29$ (m, $3 \mathrm{H}), 3.81(\mathrm{~s}, 3 \mathrm{H}), 3.78(\mathrm{~s}, 3 \mathrm{H}) .{ }^{13} \mathrm{C}$ NMR $\left(126 \mathrm{MHz}, \mathrm{CDCl}_{3}\right): \delta=135.7(\mathrm{~d}, J=3.7 \mathrm{~Hz}), 129.7(\mathrm{~d}, J=2.5 \mathrm{~Hz})$, $129.1(\mathrm{~d}, J=2.5 \mathrm{~Hz}), 123.4(\mathrm{~d}, J=8.7 \mathrm{~Hz}), 54.2(\mathrm{~d}, J=6.2 \mathrm{~Hz}) .{ }^{31} \mathrm{P}$ $\operatorname{NMR}\left(202 \mathrm{MHz}, \mathrm{CDCl}_{3}\right): \delta=21.9$.

\section{0,0-Dibutyl Se-Phenyl Phosphoroselenoate (3c)}

$188 \mathrm{mg}$ (90\% yield). ${ }^{1} \mathrm{H}$ NMR ( $\left.500 \mathrm{MHz}, \mathrm{CDCl}_{3}\right): \delta=7.66-7.62$ $(\mathrm{m}, 2 \mathrm{H}), 7.37-7.27(\mathrm{~m}, 3 \mathrm{H}), 4.18-4.02(\mathrm{~m}, 4 \mathrm{H}), 1.66-1.59(\mathrm{~m}, 4$ $\mathrm{H}), 1.42-1.34(\mathrm{~m}, 4 \mathrm{H}), 0.89(\mathrm{t}, J=7.4 \mathrm{~Hz}, 6 \mathrm{H}) .{ }^{13} \mathrm{C}$ NMR $(126$ $\left.\mathrm{MHz}, \mathrm{CDCl}_{3}\right): \delta=135.6(\mathrm{~d}, J=4.7 \mathrm{~Hz}), 129.5(\mathrm{~d}, J=2.0 \mathrm{~Hz}), 128.8$ $(\mathrm{d}, J=2.5 \mathrm{~Hz}), 124.0(\mathrm{~d}, J=8.4 \mathrm{~Hz}), 67.7(\mathrm{~d}, J=6.4 \mathrm{~Hz}), 32.2(\mathrm{~d}, J=$ 7.3 Hz), 18.8, 13.7. ${ }^{31}$ P NMR $\left(202 \mathrm{MHz}, \mathrm{CDCl}_{3}\right): \delta=18.0$. 0,0-Diisopropyl Se-Phenyl Phosphoroselenoate (3d) $168 \mathrm{mg}$ (87\% yield). ${ }^{1} \mathrm{H}$ NMR $\left(500 \mathrm{MHz}, \mathrm{CDCl}_{3}\right): \delta=7.71-7.62$ (m, $2 \mathrm{H}), 7.35-7.27(\mathrm{~m}, 3 \mathrm{H}), 4.84-4.73(\mathrm{~m}, 2 \mathrm{H}), 1.33$ (d, $J=6.2$ $\mathrm{Hz}, 6 \mathrm{H}), 1.25(\mathrm{~d}, J=6.2 \mathrm{~Hz}, 6 \mathrm{H}) .{ }^{13} \mathrm{C} \mathrm{NMR}\left(126 \mathrm{MHz} \mathrm{CDCl}_{3}\right): \delta=$ $135.3(\mathrm{~d}, J=4.9 \mathrm{~Hz}), 129.4(\mathrm{~d}, J=1.8 \mathrm{~Hz}), 128.6(\mathrm{~d}, J=2.3 \mathrm{~Hz})$, $124.7(\mathrm{~d}, J=8.3 \mathrm{~Hz}), 73.2(\mathrm{~d}, J=6.6 \mathrm{~Hz}), 24.0(\mathrm{~d}, J=3.8 \mathrm{~Hz}), 23.6$ $(\mathrm{d}, J=6.0 \mathrm{~Hz}) .{ }^{31} \mathrm{P}$ NMR $\left(202 \mathrm{MHz}, \mathrm{CDCl}_{3}\right): \delta=14.6$.

0,0-Bis(2-ethylhexyl) Se-Phenyl Phosphoroselenoate (3e)

$218 \mathrm{mg}$ (79\% yield). ${ }^{1} \mathrm{H}$ NMR $\left(500 \mathrm{MHz}, \mathrm{CDCl}_{3}\right): \delta=7.70-7.60$ (m, 2 H), 7.37-7.26 (m, 3 H), 4.08-3.92 (m, 4 H), 1.59-1.47 (m, 2
H), 1.36-1.19 (m, $16 \mathrm{H}), 0.91-0.81(\mathrm{~m}, 12 \mathrm{H}) .{ }^{13} \mathrm{C}$ NMR (126 $\left.\mathrm{MHz}, \mathrm{CDCl}_{3}\right): \delta=135.6(\mathrm{dt}, J=4.7,1.8 \mathrm{~Hz}), 129.5(\mathrm{~d}, J=1.9 \mathrm{~Hz})$, $128.7(\mathrm{~d}, J=2.3 \mathrm{~Hz}), 124.0(\mathrm{~d}, J=8.4 \mathrm{~Hz}), 70.0(\mathrm{dd}, J=7.6,2.1$ $\mathrm{Hz}), 40.1$ (d, $J=1.2 \mathrm{~Hz}), 30.0$ (d, $J=6.9 \mathrm{~Hz}), 28.9(\mathrm{~d}, J=2.2 \mathrm{~Hz})$, $23.4(\mathrm{~d}, J=5.6 \mathrm{~Hz}), 23.0,14.1,11.0 .{ }^{31} \mathrm{P}$ NMR $\left(202 \mathrm{MHz}, \mathrm{CDCl}_{3}\right)$ : $\delta=17.8$

0,0-Dibenzyl Se-Phenyl Phosphoroselenoate (3f)

$211 \mathrm{mg}\left(84 \%\right.$ yield). ${ }^{1} \mathrm{H}$ NMR $\left(500 \mathrm{MHz}, \mathrm{CDCl}_{3}\right): \delta=7.59-7.53$ (m, $2 \mathrm{H}), 7.36-7.30(\mathrm{~m}, 7 \mathrm{H}), 7.29-7.22(\mathrm{~m}, 6 \mathrm{H}), 5.12$ (qd, $J=$ 11.7, $8.7 \mathrm{~Hz}, 4 \mathrm{H}) .{ }^{13} \mathrm{C}$ NMR $\left(126 \mathrm{MHz}, \mathrm{CDCl}_{3}\right): \delta=136.0$ (d, $J=$ $4.7 \mathrm{~Hz}), 135.5(\mathrm{~d}, J=7.9 \mathrm{~Hz}), 129.7(\mathrm{~d}, J=2.2 \mathrm{~Hz}), 129.1(\mathrm{~d}, J=2.6$ $\mathrm{Hz}), 128.7,128.6,128.3,123.5(\mathrm{~d}, J=8.6 \mathrm{~Hz}), 69.3(\mathrm{~d}, J=6.1 \mathrm{~Hz})$. ${ }^{31} \mathrm{P}$ NMR $\left(202 \mathrm{MHz}, \mathrm{CDCl}_{3}\right): \delta=18.5$.

5,5-Dimethyl-2-(phenylselanyl)-1,3,2-dioxaphosphinane 2Oxide (3g)

$154 \mathrm{mg}$ (84\% yield). ${ }^{1} \mathrm{H}$ NMR (500 $\left.\mathrm{MHz}, \mathrm{CDCl}_{3}\right): \delta=7.76-7.72$ (m, $2 \mathrm{H}), 7.39-7.30$ (m, $3 \mathrm{H}), 4.15$ (dd, $J=10.6,4.3 \mathrm{~Hz}, 2 \mathrm{H}), 3.87$ (ddt, $J=24.7,11.4,1.6 \mathrm{~Hz}, 2 \mathrm{H}), 1.28(\mathrm{~s}, 3 \mathrm{H}), 0.86(\mathrm{~s}, 3 \mathrm{H}) .{ }^{13} \mathrm{C}$ NMR $\left(126 \mathrm{MHz}, \mathrm{CDCl}_{3}\right): \delta=136.0(\mathrm{~d}, J=4.7 \mathrm{~Hz}), 129.8(\mathrm{~d}, J=2.0$ $\mathrm{Hz}), 129.2(\mathrm{~d}, J=2.5 \mathrm{~Hz}), 122.0(\mathrm{~d}, J=8.0 \mathrm{~Hz}), 78.2(\mathrm{~d}, J=7.3 \mathrm{~Hz})$, $32.7(\mathrm{~d}, J=7.0 \mathrm{~Hz}), 22.3,20.6(\mathrm{~d}, J=1.0 \mathrm{~Hz}) .{ }^{31} \mathrm{P}$ NMR $(202 \mathrm{MHz}$, $\left.\mathrm{CDCl}_{3}\right): \delta=9.7$.

0,0,Se-Triphenyl Phosphoroselenoate ( $3 \mathrm{~h})$

$154 \mathrm{mg}$ (95\% yield). ${ }^{1} \mathrm{H}$ NMR (500 MHz, $\left.\mathrm{CDCl}_{3}\right): \delta=7.62-7.40$ ( $\mathrm{m}, 2 \mathrm{H}), 7.35-7.25(\mathrm{~m}, 7 \mathrm{H}), 7.20-7.16(\mathrm{~m}, 6 \mathrm{H}) .{ }^{13} \mathrm{C}$ NMR (126 $\left.\mathrm{MHz}, \mathrm{CDCl}_{3}\right): \delta=150.3(\mathrm{~d}, J=8.6 \mathrm{~Hz}), 136.3(\mathrm{~d}, J=4.8 \mathrm{~Hz}), 129.8$ $(\mathrm{d}, J=1.0 \mathrm{~Hz}), 129.6(\mathrm{~d}, J=2.5 \mathrm{~Hz}), 129.4(\mathrm{~d}, J=3.0 \mathrm{~Hz}), 125.7$ (d, $J=1.5 \mathrm{~Hz}), 122.7(\mathrm{~d}, J=9.1 \mathrm{~Hz}), 120.7(\mathrm{~d}, J=5.1 \mathrm{~Hz}) .{ }^{31} \mathrm{P}$ NMR $\left(202 \mathrm{MHz}, \mathrm{CDCl}_{3}\right): \delta=9.4$.

\section{Se-Phenyl Diphenylphosphinoselenoate (3i)}

$198 \mathrm{mg}$ (92\% yield). ${ }^{1} \mathrm{H}$ NMR (500 $\left.\mathrm{MHz}, \mathrm{CDCl}_{3}\right): \delta=7.87-7.77$ (m, $4 \mathrm{H}), 7.53-7.46(\mathrm{~m}, 4 \mathrm{H}), 7.46-7.41(\mathrm{~m}, 4 \mathrm{H}), 7.26-7.23(\mathrm{~m}, 1$ $\mathrm{H}), 7.19-7.12(\mathrm{~m}, 2 \mathrm{H}) .{ }^{13} \mathrm{C} \mathrm{NMR}\left(126 \mathrm{MHz}, \mathrm{CDCl}_{3}\right): \delta=136.4(\mathrm{~d}$, $J=3.3 \mathrm{~Hz}), 134.0(\mathrm{~s}), 133.3(\mathrm{~s}), 132.4(\mathrm{~d}, J=3.1 \mathrm{~Hz}), 131.5$ (d, $J=$ $10.6 \mathrm{~Hz}), 129.4(\mathrm{~d}, J=1.6 \mathrm{~Hz}), 128.8(\mathrm{~d}, J=2.0 \mathrm{~Hz}), 128.6(\mathrm{~d}, J=$ $13.2 \mathrm{~Hz}), 123.9(\mathrm{~d}, J=5.7 \mathrm{~Hz}) .{ }^{31} \mathrm{P}$ NMR $\left(202 \mathrm{MHz}, \mathrm{CDCl}_{3}\right): \delta=$ 20.2 .

Se-Phenyl Di-p-tolylphosphinoselenoate (3j)

$204 \mathrm{mg}$ (88\% yield). ${ }^{1} \mathrm{H}$ NMR $\left(500 \mathrm{MHz}, \mathrm{CDCl}_{3}\right): \delta=7.73-7.66$ (m, 4 H), 7.52-7.46 (m, 2 H), 7.25-7.19 (m, 5 H), 7.18-7.13 (m, 2 $\mathrm{H}), 2.37(\mathrm{~s}, 6 \mathrm{H}) .{ }^{13} \mathrm{C}$ NMR $\left(126 \mathrm{MHz}, \mathrm{CDCl}_{3}\right): \delta=142.9(\mathrm{~d}, J=3.1$ $\mathrm{Hz}), 136.4(\mathrm{~d}, J=3.3 \mathrm{~Hz}), 131.5(\mathrm{~d}, J=11.0 \mathrm{~Hz}), 131.1,130.3$, $129.42(\mathrm{~d}, J=13.6 \mathrm{~Hz}), 129.40(\mathrm{~d}, J=1.5 \mathrm{~Hz}), 128.7(\mathrm{~d}, J=1.9 \mathrm{~Hz})$, $124.5(\mathrm{~d}, J=5.6 \mathrm{~Hz}), 21.8 .{ }^{31} \mathrm{P}$ NMR $\left(202 \mathrm{MHz}, \mathrm{CDCl}_{3}\right): \delta=40.2$. Se-Phenyl Bis(3,5-dimethylphenyl)phosphinoselenoate (3k) $212 \mathrm{mg}$ (85\% yield). ${ }^{1} \mathrm{H}$ NMR (500 $\left.\mathrm{MHz}, \mathrm{CDCl}_{3}\right): \delta=7.53-7.48$ ( $\mathrm{m}, 2 \mathrm{H}), 7.45-7.37$ ( $\mathrm{m}, 4 \mathrm{H}), 7.26-7.22$ ( $\mathrm{m}, 1 \mathrm{H}), 7.19-7.14$ ( $\mathrm{m}, 2$ $\mathrm{H}), 7.12-7.07(\mathrm{~m}, 2 \mathrm{H}), 2.30(\mathrm{~s}, 12 \mathrm{H}) .{ }^{13} \mathrm{C}$ NMR $(126 \mathrm{MHz}$, $\left.\mathrm{CDCl}_{3}\right): \delta=138.2(\mathrm{~d}, J=13.9 \mathrm{~Hz}), 136.4(\mathrm{~d}, J=3.2 \mathrm{~Hz}), 134.0(\mathrm{~d}$, $J=3.2 \mathrm{~Hz}), 133.0,129.2(\mathrm{~d}, J=1.5 \mathrm{~Hz}), 128.9(\mathrm{~d}, J=10.5 \mathrm{~Hz})$, $128.7(\mathrm{~d}, J=1.9 \mathrm{~Hz}), 124.3(\mathrm{~d}, J=5.7 \mathrm{~Hz}), 21.3 .{ }^{31} \mathrm{P}$ NMR $(202$ $\left.\mathrm{MHz}, \mathrm{CDCl}_{3}\right): \delta=41.2$.

Se-Phenyl Di(naphthalen-2-yl)phosphinoselenoate (31) $228 \mathrm{mg}$ (83\% yield). ${ }^{1} \mathrm{H}$ NMR (500 MHz, $\left.\mathrm{CDCl}_{3}\right): \delta=8.45$ (d, $J=$ $15.0 \mathrm{~Hz}, 2 \mathrm{H}), 7.93-7.82(\mathrm{~m}, 8 \mathrm{H}), 7.61-7.51(\mathrm{~m}, 6 \mathrm{H}), 7.22-7.15$ $(\mathrm{m}, 1 \mathrm{H}), 7.14-7.06(\mathrm{~m}, 2 \mathrm{H}) .{ }^{13} \mathrm{C}$ NMR $\left(126 \mathrm{MHz}, \mathrm{CDCl}_{3}\right): \delta=$ $136.4(\mathrm{~d}, J=3.2 \mathrm{~Hz}), 134.9$ (d, $J=2.6 \mathrm{~Hz}), 133.7(\mathrm{~d}, J=9.6 \mathrm{~Hz})$, $132.5(\mathrm{~d}, J=14.4 \mathrm{~Hz}), 131.0,130.25,129.4(\mathrm{~d}, J=1.5 \mathrm{~Hz}), 129.2$, $128.8(\mathrm{~d}, J=1.9 \mathrm{~Hz}), 128.5(\mathrm{~d}, J=13.3 \mathrm{~Hz}), 127.9,127.1,126.1(\mathrm{~d}$, $J=12.1 \mathrm{~Hz}), 123.9(\mathrm{~d}, J=5.7 \mathrm{~Hz}) .{ }^{31} \mathrm{P}$ NMR $\left(202 \mathrm{MHz}, \mathrm{CDCl}_{3}\right): \delta=$ 39.8 .

O-Ethyl Se-Phenyl Phenylphosphonoselenoate ( $3 \mathrm{~m}$ )

$156 \mathrm{mg}$ (80\% yield). ${ }^{1} \mathrm{H}$ NMR (500 MHz, $\mathrm{CDCl}_{3}$ ): $\delta=7.54-7.47$ 
(m, $2 \mathrm{H}), 7.40-7.35(\mathrm{~m}, 1 \mathrm{H}), 7.28-7.16(\mathrm{~m}, 5 \mathrm{H}), 7.10-7.04(\mathrm{~m}, 2$ $\mathrm{H}), 4.32-4.18(\mathrm{~m}, 2 \mathrm{H}), 1.31(\mathrm{t}, J=7.1 \mathrm{~Hz}, 3 \mathrm{H}) .{ }^{13} \mathrm{C}$ NMR $(126$ $\left.\mathrm{MHz}, \mathrm{CDCl}_{3}\right): \delta=150.5(\mathrm{~d}, J=3.6 \mathrm{~Hz}), 133.4,132.5(\mathrm{~d}, J=3.3 \mathrm{~Hz})$, $132.3,131.1(\mathrm{~d}, J=11.0 \mathrm{~Hz}), 129.3(\mathrm{~d}, J=2.1 \mathrm{~Hz}), 128.8(\mathrm{~d}, J=2.5$ $\mathrm{Hz}), 128.2(\mathrm{~d}, J=14.9 \mathrm{~Hz}), 124.3(\mathrm{~d}, J=6.6 \mathrm{~Hz}), 62.7,16.3 .{ }^{31} \mathrm{P}$ $\operatorname{NMR}\left(202 \mathrm{MHz}, \mathrm{CDCl}_{3}\right): \delta=38.4$.

6-(Phenylselanyl)dibenzo[c,e][1,2]oxaphosphinine 6-0xide (3n)

$192 \mathrm{mg}$ (86\% yield). ${ }^{1} \mathrm{H}$ NMR (500 $\mathrm{MHz}, \mathrm{CDCl}_{3}$ ): $\delta=7.90-7.83$ (m, $1 \mathrm{H}), 7.77-7.73(\mathrm{~m}, 1 \mathrm{H}), 7.66-7.60(\mathrm{~m}, 2 \mathrm{H}), 7.46$ (tdd, $J=$ 7.5, 3.6, $1.0 \mathrm{~Hz}, 1 \mathrm{H}), 7.33-7.29(\mathrm{~m}, 1 \mathrm{H}), 7.25-7.20(\mathrm{~m}, 2 \mathrm{H})$, 7.17-7.12 (m, $3 \mathrm{H}), 7.01-6.97$ (m, $2 \mathrm{H}) .{ }^{13} \mathrm{C}$ NMR (126 MHz, $\left.\mathrm{CDCl}_{3}\right): \delta=150.5(\mathrm{~d}, J=9.9 \mathrm{~Hz}), 137.1(\mathrm{~d}, J=3.6 \mathrm{~Hz}), 136.0(\mathrm{~d}, J=$ $7.5 \mathrm{~Hz}), 133.8(\mathrm{~d}, J=2.8 \mathrm{~Hz}), 130.6(\mathrm{~d}, J=11.0 \mathrm{~Hz}), 129.19(\mathrm{~d}, J=$ $2.5 \mathrm{~Hz}), 129.13$ (d, $J=2.9 \mathrm{~Hz}), 128.5(\mathrm{~d}, J=14.9 \mathrm{~Hz}), 126.6$, $125.73,125.0(\mathrm{~d}, J=1.0 \mathrm{~Hz}), 124.8,123.3(\mathrm{~d}, J=11.2 \mathrm{~Hz}), 122.1$ (d, $J=7.2 \mathrm{~Hz}), 122.0$ (d, $J=11.7 \mathrm{~Hz}), 120.2$ (d, $J=7.0 \mathrm{~Hz}) .{ }^{31} \mathrm{P}$ NMR $\left(202 \mathrm{MHz}, \mathrm{CDCl}_{3}\right): \delta=31.2$.

Se-Benzyl 0,0-Diethyl Phosphoroselenoate (3o)

$136 \mathrm{mg}$ (74\% yield). The ${ }^{1} \mathrm{H}$ NMR $\left(500 \mathrm{MHz}, \mathrm{CDCl}_{3}\right): \delta=7.41-$ $7.32(\mathrm{~m}, 2 \mathrm{H}), 7.32-7.27$ ( $\mathrm{m}, 2 \mathrm{H}), 7.26-7.20(\mathrm{~m}, 1 \mathrm{H}), 4.16-3.98$ $(\mathrm{m}, 6 \mathrm{H}), 1.30(\mathrm{td}, J=7.1,0.7 \mathrm{~Hz}, 6 \mathrm{H}) .{ }^{13} \mathrm{C}$ NMR $(126 \mathrm{MHz}$, $\left.\mathrm{CDCl}_{3}\right): \delta=138.5(\mathrm{~d}, J=4.7 \mathrm{~Hz}), 129.0,128.7,127.5,63.5(\mathrm{~d}, J=$ $5.5 \mathrm{~Hz}), 29.5(\mathrm{~d}, J=4.6 \mathrm{~Hz}), 16.0(\mathrm{~d}, J=7.5 \mathrm{~Hz}) .{ }^{31} \mathrm{P}$ NMR $(202$ $\left.\mathrm{MHz}, \mathrm{CDCl}_{3}\right): \delta=20.2$.

Se-Benzyl 0,0-Diphenyl Phosphoroselenoate (3p)

$193 \mathrm{mg}$ (80\% yield). ${ }^{1} \mathrm{H}$ NMR $\left(500 \mathrm{MHz}, \mathrm{CDCl}_{3}\right): \delta=7.28-7.24$ $(\mathrm{m}, 4 \mathrm{H}), 7.16-7.12(\mathrm{~m}, 11 \mathrm{H}), 4.08(\mathrm{~d}, J=12.6 \mathrm{~Hz}, 2 \mathrm{H}) .{ }^{13} \mathrm{C}$ NMR $\left(126 \mathrm{MHz}, \mathrm{CDCl}_{3}\right): \delta=137.2(\mathrm{~d}, J=6.0 \mathrm{~Hz}), 129.9(\mathrm{~d}, J=1.3 \mathrm{~Hz})$, 129.2, 128.8, 127.7, 125.8 (d, $J=1.7 \mathrm{~Hz}), 121.0$ (d, $J=4.9 \mathrm{~Hz})$, $120.4,30.9(\mathrm{~d}, J=4.8 \mathrm{~Hz}) .{ }^{31} \mathrm{P}$ NMR $\left(202 \mathrm{MHz}, \mathrm{CDCl}_{3}\right): \delta=13.7$.

Se-Benzyl Diphenylphosphinoselenoate (3q)

$174 \mathrm{mg}$ (78\% yield). ${ }^{1} \mathrm{H}$ NMR $\left(500 \mathrm{MHz}, \mathrm{CDCl}_{3}\right): \delta=7.94-7.78$ (m, $4 \mathrm{H}), 7.55-7.49(\mathrm{~m}, 2 \mathrm{H}), 7.48-7.35(\mathrm{~m}, 4 \mathrm{H}), 7.20-7.10(\mathrm{~m}, 5$ $\mathrm{H}), 4.07(\mathrm{~d}, J=8.3 \mathrm{~Hz}, 2 \mathrm{H}) .{ }^{13} \mathrm{C} \mathrm{NMR}\left(126 \mathrm{MHz}, \mathrm{CDCl}_{3}\right): \delta=137.7$ $(\mathrm{d}, J=4.4 \mathrm{~Hz}), 134.6,133.8,132.4(\mathrm{~d}, J=3.1 \mathrm{~Hz}), 131.4(\mathrm{~d}, J=10.9$ $\mathrm{Hz}), 129.2,128.8,128.7(\mathrm{~d}, J=5.2 \mathrm{~Hz}), 127.3,28.4(\mathrm{~d}, J=2.5 \mathrm{~Hz})$. ${ }^{31} \mathrm{P} \operatorname{NMR}\left(202 \mathrm{MHz}, \mathrm{CDCl}_{3}\right): \delta=40.0$.

\section{6-(Benzylselanyl)dibenzo[c,e][1,2] $]$ oxaphosphinine 6-0xide} (3r)

$165 \mathrm{mg}$ (71\% yield). ${ }^{1} \mathrm{H}$ NMR $\left(500 \mathrm{MHz}, \mathrm{CDCl}_{3}\right): \delta=7.95-7.84$ ( $\mathrm{m}, 3 \mathrm{H}), 7.70-7.65$ ( $\mathrm{m}, 1 \mathrm{H}), 7.49$ (tdd, $J=7.6,3.6,0.9 \mathrm{~Hz}, 1 \mathrm{H}$ ), 7.38-7.33 (m, $1 \mathrm{H}), 7.29-7.19(\mathrm{~m}, 6 \mathrm{H}), 7.11-7.05(\mathrm{~m}, 1 \mathrm{H})$, 4.24-4.09 (m, $2 \mathrm{H}) .{ }^{13} \mathrm{C} \mathrm{NMR}\left(126 \mathrm{MHz}, \mathrm{CDCl}_{3}\right): \delta=149.4(\mathrm{~d}, J=$ $9.8 \mathrm{~Hz}), 137.7(\mathrm{~d}, J=4.4 \mathrm{~Hz}), 135.6(\mathrm{~d}, J=7.6 \mathrm{~Hz}), 133.8(\mathrm{~d}, J=2.7$ $\mathrm{Hz}), 130.8,130.4(\mathrm{~d}, J=11.9 \mathrm{~Hz}), 129.2,128.7(\mathrm{t}, J=7.5 \mathrm{~Hz})$, 127.8, 127.5, 126.8, $125.2(\mathrm{~d}, J=1.1 \mathrm{~Hz}), 125.1(\mathrm{~d}, J=0.6 \mathrm{~Hz})$, $123.8(\mathrm{~d}, J=10.9 \mathrm{~Hz}), 122.3(\mathrm{~d}, J=12.3 \mathrm{~Hz}), 120.6(\mathrm{~d}, J=6.6 \mathrm{~Hz})$, $29.2(\mathrm{~d}, J=3.7 \mathrm{~Hz}) .{ }^{3} \mathrm{P}$ NMR $\left(202 \mathrm{MHz}, \mathrm{CDCl}_{3}\right): \delta=33.6$.

0,0-Diethyl S-Phenyl Phosphorothioate (3s)

$84 \mathrm{mg}$ (57\% yield). ${ }^{1} \mathrm{H}$ NMR $\left(500 \mathrm{MHz}, \mathrm{CDCl}_{3}\right): \delta=7.59-7.52(\mathrm{~m}$, $2 \mathrm{H}), 7.37-7.31(\mathrm{~m}, 3 \mathrm{H}), 4.25-4.12(\mathrm{~m}, 4 \mathrm{H}), 1.30(\mathrm{td}, J=7.1,0.9$ $\mathrm{Hz}, 6 \mathrm{H}) .{ }^{13} \mathrm{C}$ NMR $\left(126 \mathrm{MHz}, \mathrm{CDCl}_{3}\right): \delta=134.6(\mathrm{~d}, J=5.2 \mathrm{~Hz})$, $129.5(\mathrm{~d}, J=2.2 \mathrm{~Hz}), 129.1(\mathrm{~d}, J=2.8 \mathrm{~Hz}), 126.7(\mathrm{~d}, J=7.2 \mathrm{~Hz})$, 64.2 (d, $J=6.2 \mathrm{~Hz}), 16.1$ (d, $J=7.2 \mathrm{~Hz}) .{ }^{31} \mathrm{P}$ NMR $(202 \mathrm{MHz}$, $\left.\mathrm{CDCl}_{3}\right): \delta=22.9$.

$S$-Phenyl Diphenylphosphinothioate (3t)

$124 \mathrm{mg}$ ( $66 \%$ yield). ${ }^{1} \mathrm{H}$ NMR (500 $\left.\mathrm{MHz}, \mathrm{CDCl}_{3}\right): \delta=7.88-7.81$ (m, $4 \mathrm{H}), 7.53-7.48(\mathrm{~m}, 2 \mathrm{H}), 7.47-7.41(\mathrm{~m}, 6 \mathrm{H}), 7.26-7.22(\mathrm{~m}, 1$ $\mathrm{H}), 7.22-7.17(\mathrm{~m}, 2 \mathrm{H}) .{ }^{13} \mathrm{C} \mathrm{NMR}\left(126 \mathrm{MHz}, \mathrm{CDCl}_{3}\right): \delta=135.5(\mathrm{~d}$, $J=3.9 \mathrm{~Hz}), 133.1,132.4(\mathrm{~d}, J=3.0 \mathrm{~Hz}), 131.7(\mathrm{~d}, J=10.2 \mathrm{~Hz})$, $129.2(\mathrm{~d}, J=1.7 \mathrm{~Hz}), 129.0(\mathrm{~d}, J=2.2 \mathrm{~Hz}), 128.6(\mathrm{~d}, J=13.1 \mathrm{~Hz})$, $126.3(\mathrm{~d}, J=5.2 \mathrm{~Hz}) \cdot{ }^{31} \mathrm{P}$ NMR $\left(202 \mathrm{MHz}, \mathrm{CDCl}_{3}\right): \delta=41.3$.

6-(Phenylthio)dibenzo[c,e][1,2]oxaphosphinine 6-Oxide (3u) $135 \mathrm{mg}$ (69\% yield). ${ }^{1} \mathrm{H}$ NMR (500 MHz, $\mathrm{CDCl}_{3}$ ): $\delta=7.96-7.89$ $(\mathrm{m}, 1 \mathrm{H}), 7.86-7.81(\mathrm{~m}, 1 \mathrm{H}), 7.75(\mathrm{dd}, J=7.8,1.6 \mathrm{~Hz}, 1 \mathrm{H}), 7.70-$ 7.65 (m, $1 \mathrm{H}), 7.50$ (tdd, $J=7.5,3.6,1.0 \mathrm{~Hz}, 1 \mathrm{H}), 7.38-7.34(\mathrm{~m}, 1$ H), 7.28-7.16 (m, $5 \mathrm{H}), 7.12-7.06$ ( $\mathrm{m}, 2 \mathrm{H}) .{ }^{13} \mathrm{C}$ NMR (126 MHz, $\left.\mathrm{CDCl}_{3}\right): \delta=150.6(\mathrm{~d}, J=9.6 \mathrm{~Hz}), 136.4(\mathrm{~d}, J=7.3 \mathrm{~Hz}), 136.1(\mathrm{~d}, J=$ $4.3 \mathrm{~Hz}), 133.8$ (d, $J=2.6 \mathrm{~Hz}), 130.9$ (d, $J=10.2 \mathrm{~Hz}), 130.6,129.4$ $(\mathrm{d}, J=3.1 \mathrm{~Hz}), 129.1(\mathrm{~d}, J=2.6 \mathrm{~Hz}), 128.5(\mathrm{~d}, J=14.9 \mathrm{~Hz}), 125.4$, 124.7, 124.3 (d, $J=4.9 \mathrm{~Hz}), 123.3(\mathrm{~d}, J=11.5 \mathrm{~Hz}), 121.9$ (d, $J=$ $11.6 \mathrm{~Hz}), 120.1$ (d, $J=7.1 \mathrm{~Hz}) .{ }^{31} \mathrm{P}$ NMR $\left(202 \mathrm{MHz}, \mathrm{CDCl}_{3}\right): \delta=$ 34.2 .

(15) Zhang, X.; Wang, C.; Jiang, H.; Sun, L. Chem. Commun. 2018, 54, 8781. 